\title{
Densely Interleaved Arrays for Dual-Tone Transmitters
}

\author{
Student Submission
}

\author{
Anton Atanasov, Mark S. Oude Alink, and Frank E. van Vliet \\ Integrated Circuit Design, University of Twente, Enschede, The Netherlands \\ Email: a.n.atanasov@utwente.nl
}

\begin{abstract}
Power amplifier nonlinearity introduces intermodulation distortion when transmitting dual-tone signals from an antenna array. To reduce distortion levels and increase output power for dual-tone systems we propose to densely interleave two antenna arrays without increasing the antenna aperture. Each array is excited with a different tone, resulting in a single-tone input for the power amplifiers, thereby reducing intermodulation distortion. In order to compare the performance of the densely interleaved array with a regular, planar array, when excited by two-tone signals, a procedure for evaluating the S-parameters matrix of an interleaved dipole array is presented. Simulations with a realistic RF power amplifier model show 13 to $31 \mathrm{~dB}$ intermodulation reduction for the same output power and total antenna aperture.
\end{abstract}

Index Terms - phased arrays, mutual coupling, intermodulation, nonlinearity, interleaved arrays

\section{INTRODUCTION}

The power and spectral efficiency of any transmitting system is directly affected by the linearity of the power amplifier (PA) in use. Achieving high linearity is a challenging design task, making it an active area of research [1]-[4]. Some of the more common techniques include adaptive feedforward linearization [5] and predistortion [6] to achieve better distortion performance. This allows the PA to operate at higher output powers, increasing its efficiency. Such an improvement is beneficial in power sensitive areas such as mobile radio, MIMO systems and radar applications.

The linearization task is further complicated when the nonlinear PA is driven by multiple input frequencies, or tones, which leads to intermodulation distortion (IMD) that causes unwanted spectrum spreading and power loss in the desired output tones. The IMD caused by multi-tone inputs cannot always be suppressed using the linearization techniques that work for single-tone systems. One creative approach to reduce the IMD caused by multi-tone input signals is outlined in [7].

In this work we propose to resolve part of the intermodulation problem by replacing the last stage of a dual-tone transmitter (PA and antenna element) with a pair of single-tone transmitters. The physical separation reduces the intermodulation effects and by densely interleaving the antenna elements, the total antenna aperture does not need to be increased. If we adapt the power level of the individual PAs, also the total dissipation does not increase, and effectively everything behaves identical in terms of desired signal transmission, but now at a much lower IMD level.
The problem that does arise, and is investigated in this paper, is whether the increased mutual coupling, due to the lower element-to-element spacing, does not give rise to increased reverse IMD [8], which could render the concept useless.

In order to do so, we investigate the case of an array of densely interleaved dipoles. In section II, the mutual coupling between these dipoles is established in a theoretical way for thin dipoles, resulting in a mutual impedance matrix $[Z]$. In section III we establish a simple non-linear amplifier model that will be used for the reference case (a regular array with two tones presented at the input of every amplifier) and for the proposed case (a densely interleaved double array of the same size as the regular array, with a separate amplifier per tone). Combining the mutual-coupling matrix with the amplifiers, we will finally evaluate the IMD of the two solutions, quantifying the benefit from this hardware split. Finally, we present our conclusions in section IV.

\section{Array Mutual Coupling}

The mutual coupling S-matrix of an antenna array is constructed from the self and mutual impedances of all interacting antenna elements within the array, excluding a ground plane and terminated with the system characteristic impedance. There exist several dedicated numerical solving techniques based on, for example, the Hellén [9] and the Pocklington integral methods [10], as well as multiple generalized solvers based on finite element, method of moments and others [11] [13]. While these techniques offer high accuracy, their numerical nature requires strict convergence criteria, potentially long computation times and little analytical insight. Instead, an approximate analytical method has been chosen here, based on the induced electromotive force (I-EMF) method [14], which assumes a sinusoidal current distribution along the dipole length [15] in order to simplify the calculations [16], allowing for easy implementation. In addition, the limitations of the method are evident from its derivation, removing guesswork about its accuracy.

The I-EMF method offers a set of closed-form expressions, which assume perfectly conducting thin dipoles and are not subject to convergence criteria. The simplifying assumption that the mutual impedance of an array can be constructed from a linear combination of isolated dipole pairs provides a good approximation for simple geometries [17], [18]. Based on this, 
the I-EMF method is used to generate coupling matrices for different normal and interleaved array configurations.

The mutual impedance [19] for a given antenna array of $N$ elements is

$$
\left[\begin{array}{c}
V_{1} \\
\vdots \\
V_{N}
\end{array}\right]=\left[\begin{array}{ccc}
Z_{11} & Z_{12} & \cdots \\
\vdots & \ddots & \\
Z_{N 1} & & Z_{N N}
\end{array}\right]\left[\begin{array}{c}
I_{1} \\
\vdots \\
I_{N}
\end{array}\right]
$$

where

$$
\left.Z_{i j} \triangleq \frac{V_{i}}{I_{j}}\right|_{I_{i \neq j}=0}
$$

is the mutual impedance for a given pair when all other antennas are left unexcited. The antenna reciprocity theorem [20] states that the coupling between two antennas is symmetric, implying that the mutual impedance is symmetric as well.

Equations (1) and (2) can be summarized as

$$
Z_{n}=\frac{V_{n}}{I_{n}}=Z_{n n}+Z_{n 1}\left(\frac{I_{1}}{I_{n}}\right)+Z_{n 2}\left(\frac{I_{2}}{I_{n}}\right)+\ldots
$$

where $Z_{n}$ is the driving-point, or input, impedance for a given antenna where $n=1 \ldots N$. The mutual impedances and the ratio of the driving currents form a weighted sum. The driving currents also contain the phase offsets for a given scan angle. When the array operates in broadside all the drive currents are in phase and so the weights are uniform. Changing the scan angle adjusts the weights, which directly affects the input impedance of all antennas. Fig. 1 shows the mutual impedance between two parallel, identical dipoles as a function of spacing $d$. As the spacing increases, the mutual impedance effect decreases. In Fig. 2 the two dipoles are in a collinear arrangement, that is side by side, and the mutual impedance is evaluated as a function of sideway offset $s$. The mutual impedance is much weaker in the latter arrangement, due to the properties of the dipoles. Both figures follow the results of [14], with the exception of the phase plots which have been unwrapped for clearer representation.

For an array of $N$ elements, there are $N \times N$ self and mutual interactions which must be computed. However, for an array of identical elements, and taking advantage of the symmetry property, the number of computations for an arbitrary array can be reduced to

$$
T_{n}=\left\lceil\frac{N \cdot(N-1)+1}{2}\right\rceil
$$

where $\lceil\cdot\rceil$ is the ceiling function. The resulting multiport impedance matrix is converted to an S-parameter matrix using the following generalized relation [21]

$$
[S]=\left[G_{0}\right] \cdot\left([Z]-\left[Z_{0}^{*}\right]\right) \cdot\left([Z]-\left[Z_{0}\right]\right)^{-1} \cdot\left[G_{0}\right]^{-1},
$$

where

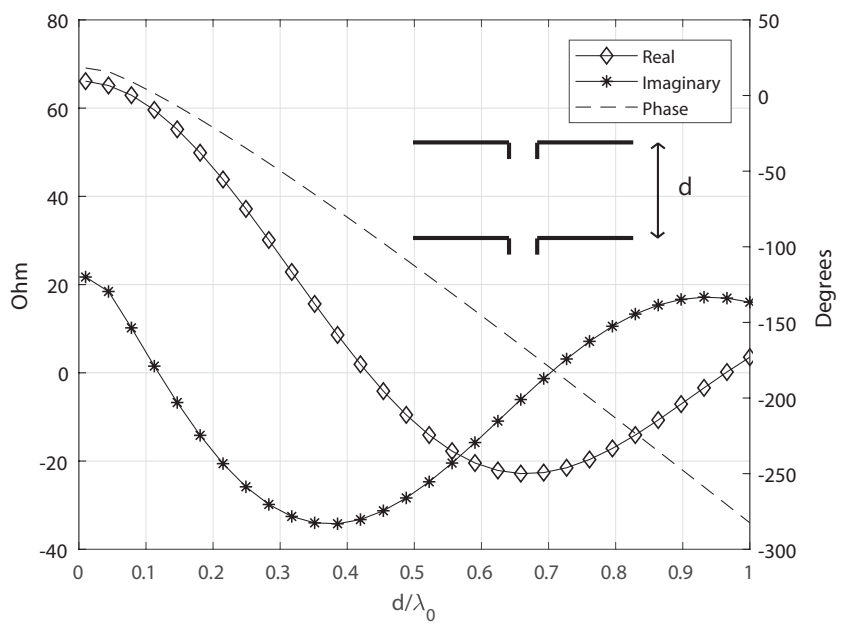

Fig. 1. Mutual impedance between two identical parallel $\lambda / 2$ dipoles as a function of spacing $d$ at resonance. As $d \rightarrow 0$, the mutual impedance converges to the dipole's self-impedance.

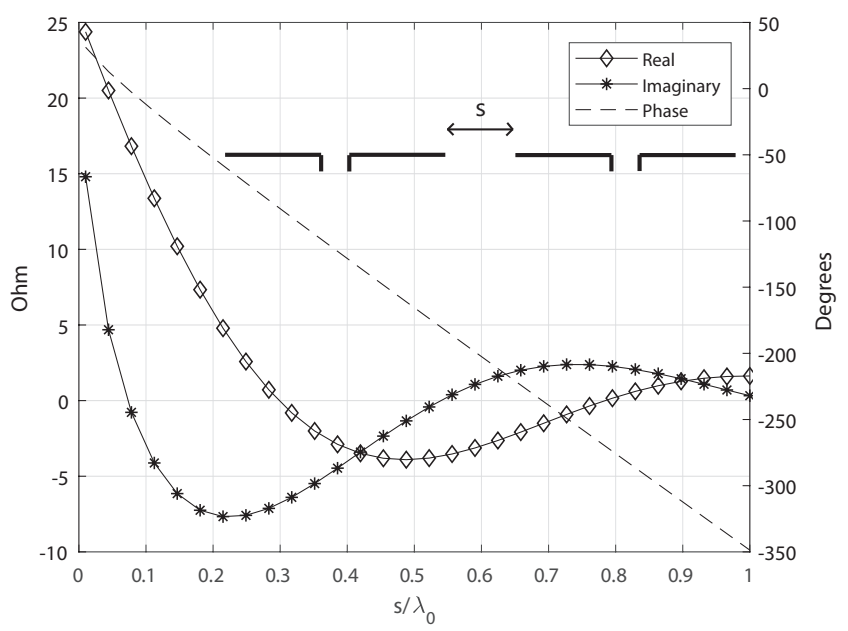

Fig. 2. Mutual impedance between two identical collinear dipoles as a function of sideway offset $s$ at resonance. As it can be seen, the mutual impedance decreases faster than in the parallel arrangement.

$$
\begin{aligned}
& {\left[Z_{0}\right] \triangleq \operatorname{diag}\left\{Z_{01}, \ldots, Z_{0 n}, \ldots, Z_{0 N}\right\}} \\
& {\left[G_{0}\right] \triangleq \operatorname{diag}\left\{g_{01}, \ldots, g_{0 n}, \ldots, g_{0 N}\right\}}
\end{aligned}
$$

are diagonal matrices, where each term is related to a given port impedance $Z_{0 n}$, in our case defined as $50 \Omega$, and

$$
g_{0 n}=\frac{1}{\sqrt{\mathfrak{R}\left\{Z_{0 n}\right\}}} .
$$

Fig. 3 shows the plot of the S-parameters for two identical dipoles, both excited by the same normalized frequency, spaced at $d=0.25 \lambda_{0} . S_{11}$ represents the behavior of a thin dipole antenna with radius of $1 \mathrm{~mm}$, while $S_{12}$ shows the mutual coupling between the two dipoles. As the normalized spacing $d / \lambda_{0}$ increases between the two dipoles, the mutual coupling decreases. Fig. 4 shows the S-parameter matrix for 


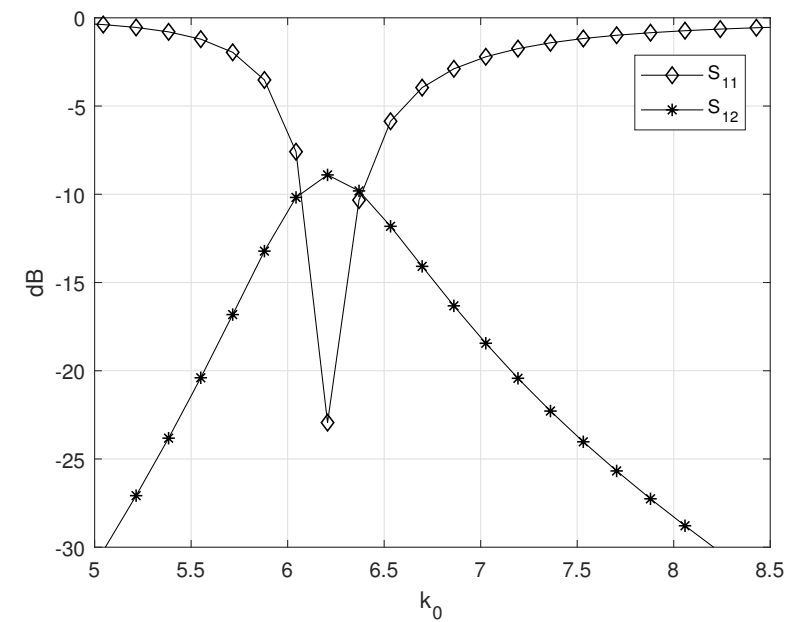

Fig. 3. $S_{11}$ and $S_{12}$ parameters vs normalized wavenumber $k_{0}$ at $d=0.25 \lambda_{0}$ for two parallel $\lambda / 2$ dipoles. The dipoles resonate at $k_{0}=2 \pi$.

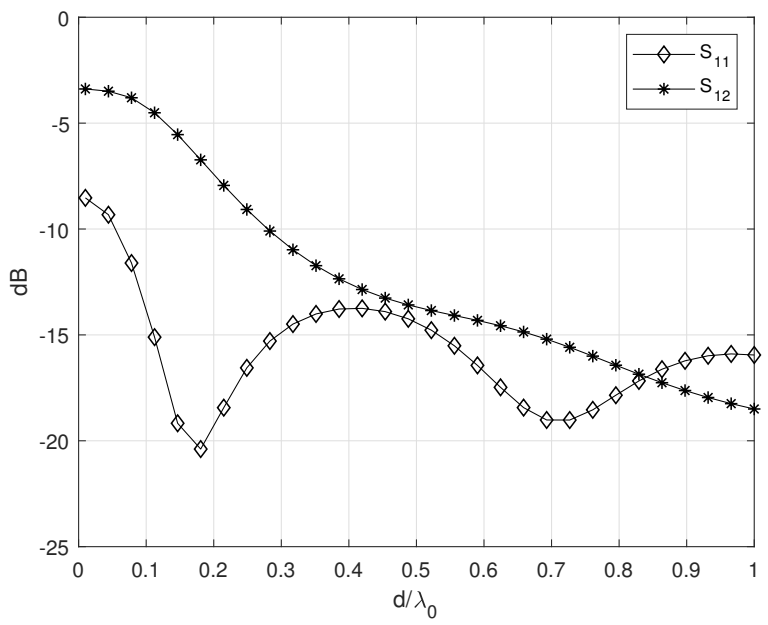

Fig. 4. $S_{11}$ and $S_{12}$ parameters vs normalized spacing $d / \lambda_{0}$ for two parallel $\lambda / 2$ dipoles.

two identical dipoles driven at resonance as a function of increasing $d / \lambda_{0}$. In the case of a two-dipole array, $S_{11}$ has a very low mutual coupling at $0.177 \lambda_{0}$, despite the two dipole's proximity.

The accuracy of the I-EMF method applied to an array is compared against the results of the 4NEC2 numerical electromagnetic simulation software [22], which uses a method of moments based solver. A pair of perfectly conducting $\lambda / 2$ dipoles with a radius of $1 \mathrm{~mm}$ in free space are simulated at multiple separations. From Fig. 4 it is clear that the mutual coupling decreases with increased spacing. Fig. 5 shows a comparison between $4 \mathrm{NEC} 2$ and the I-EMF method in the case of two perfectly conducting, parallel $\lambda / 2$ dipoles evaluated at several spacings. The I-EMF method produces reasonably similar results to those of its numerical counterpart, as long as the diameter of the dipoles is kept small.

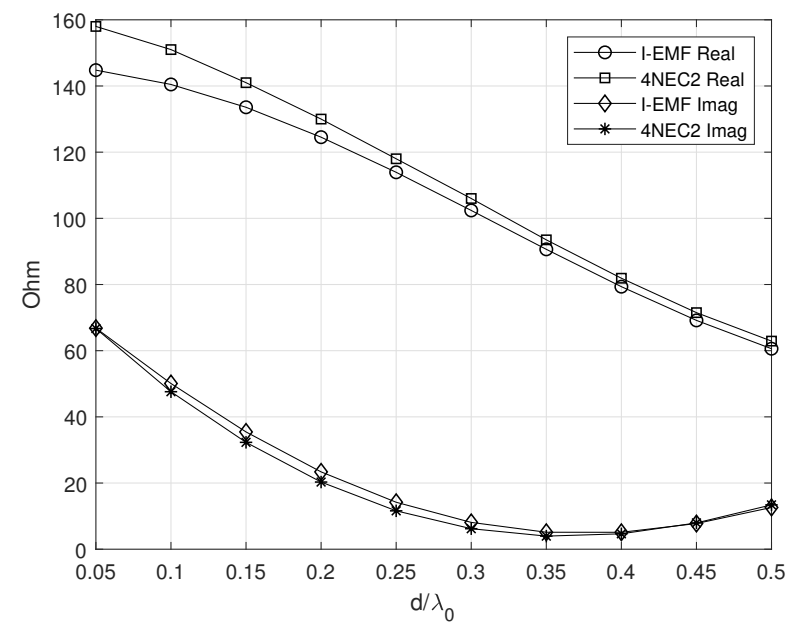

Fig. 5. Input impedance, $Z_{1}=Z_{11}+Z_{12}$, of two parallel $\lambda / 2$ dipoles as a function of $d / \lambda$ spacing

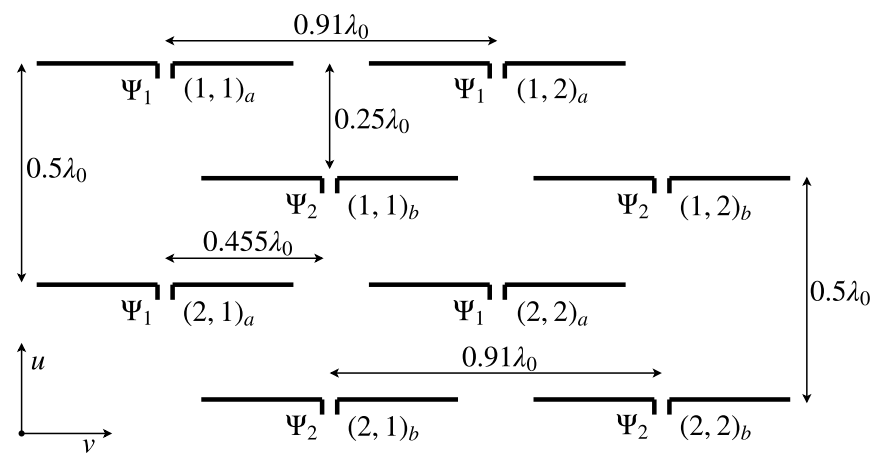

Fig. 6. Interleaved array layout, consisting of two planar arrays with parallel spacing of $0.5 \lambda_{0}$ in the $u$ direction and collinear spacing of $0.91 \lambda_{0}$ in the $v$ direction with an offset of $0.25 \lambda_{0}$ and $0.455 \lambda_{0}$. Each dipole is indexed and driven by a tone, either $\Psi_{1}$ or $\Psi_{2}$

\section{PA Simulations}

Using the I-EMF method the self and mutual impedances as a function of frequency are evaluated for a planar, regular $3 \times 3$ array with $0.5 \lambda_{0}$ spacing in the parallel direction $u$ and $0.91 \lambda_{0}$ spacing in the collinear direction $v$, making use of the directional cosine notation. The same is done for an interleaved planar array consisting of two planar, regular $3 \times 3$ arrays (operating at a single tone each) offset by $0.25 \lambda_{0}$ and $0.455 \lambda_{0}$ in the $u$ and $v$ directions, respectively. Fig. 6 shows the interleaved array layout together with the defined $u$ and $v$ directions. Each dipole is assigned an index, indicating its relative position within the array. Those with the same index are treated as a pair, with each dipole operating at a different tone, either $\Psi_{1}$ or $\Psi_{2}$. This is done for simpler comparison with the performance of the regular array. The array spacings are chosen such that the I-EMF approximation gives reliable estimates.

The IMD performance of the interleaved array is compared to that of the single planar array in Keysight's ADS. The PA used in the simulations is a Class A one [23], with a center 


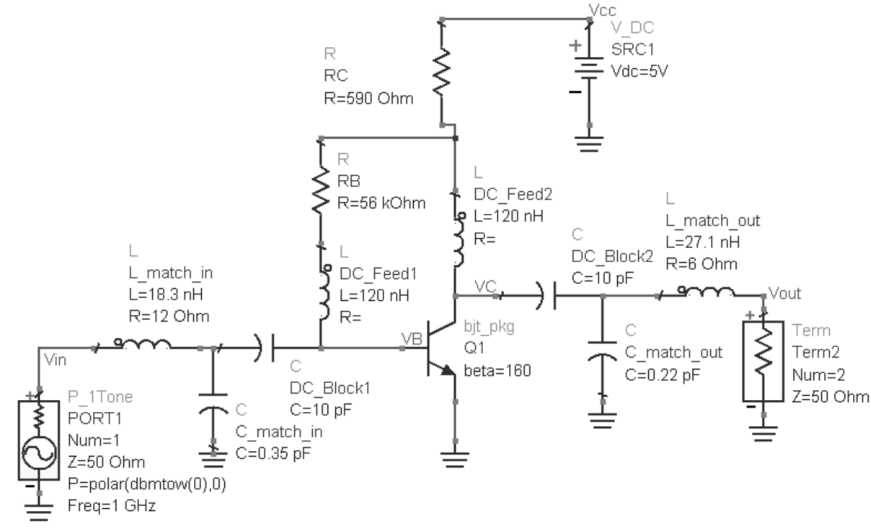

(a) Class A PA topology

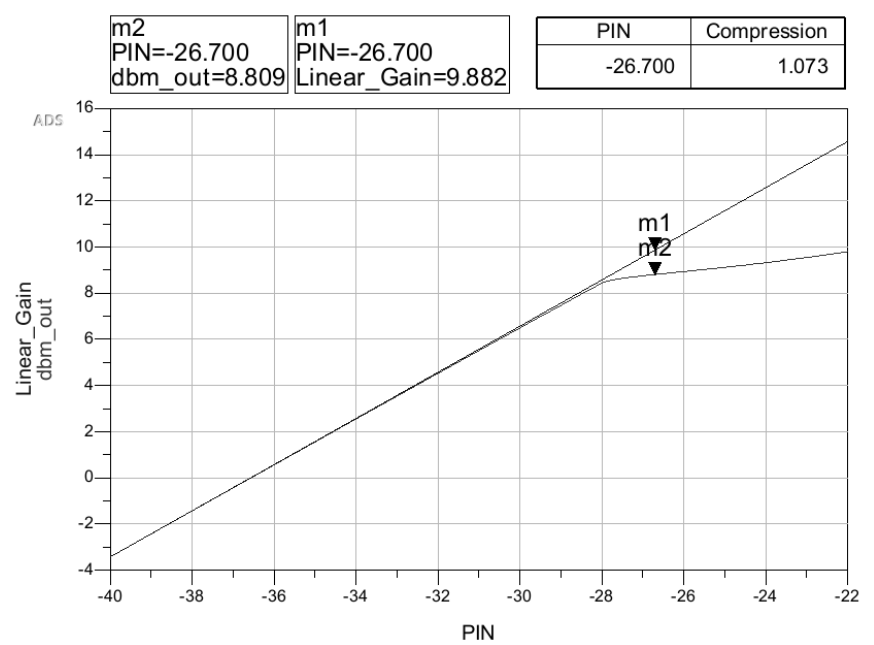

(b) PA gain versus input power $P_{i n}$

Fig. 7. (a) Topology of the Class A PA used in the ADS simulations (b) Gain of the example PA as a function of input power $P_{i n}$. The $1 \mathrm{~dB}$ compression point is at $-26.7 \mathrm{dBm} . m 1=9.9 \mathrm{dBm}$ and $m 2=8.8 \mathrm{dBm}$

frequency of $1.9 \mathrm{GHz}$ and a $1 \mathrm{~dB}$ compression point of -26.7 $\mathrm{dBm}$. The BJT is modelled with $\beta=160,320 \mathrm{pH}$ inductance on all leads and $120 \mathrm{fF}$ package capacitance between the baseemitter and collector-emitter junctions. Fig. 7 shows the PA's topology simulated in ADS and a plot of its gain versus input power. In the following simulations the frequency spacing between the tones is $10 \mathrm{MHz}$ and the power level of each input tone is $-29.7 \mathrm{dBm}$, giving a combined input power equal to the $1 \mathrm{~dB}$ compression point of $-26.7 \mathrm{dBm}$.

First, a direct comparison is made between a single PA driving two tones into a single $\lambda / 2$ dipole and two PAs driving a single tone at $-29.7 \mathrm{dBm}$ each into two interleaved $\lambda / 2$ dipoles, with the same offsets as described earlier. Fig. 8 shows the output power spectrum of the single PA driving two tones. Both output tones have similar power levels of around $9 \mathrm{dBm}$ and the spurious signal level, $\Delta$, defined as the difference between the strongest active tone and the strongest IM3 component, is $-28.1 \mathrm{dBc}$. Next, Fig. 9 shows the output power spectrum of each PA driving a single tone into each $\lambda / 2$ dipole of the interleaved pair. Due to the spacing of the dipoles, only part of the power of each tone couples to each adjacent

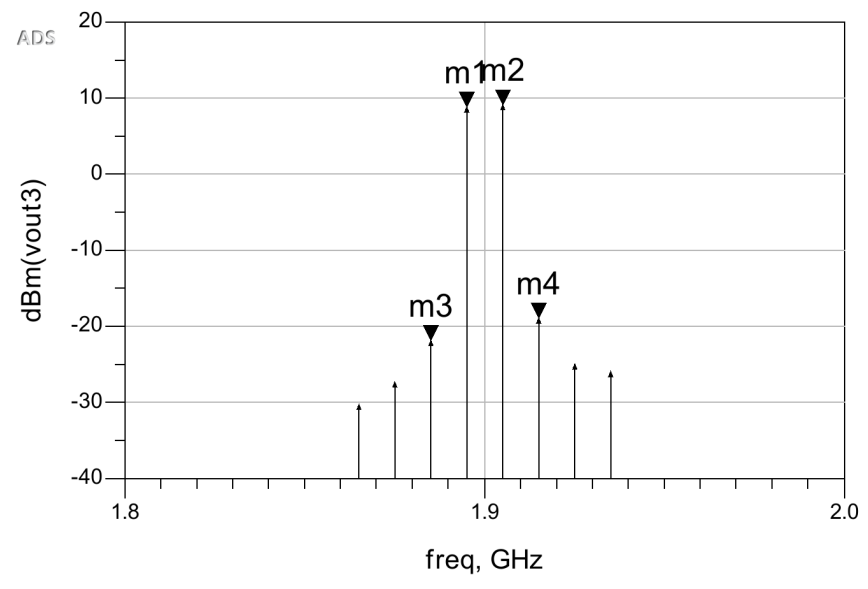

Fig. 8. PA output power spectrum for single dipole antenna. The PA is driven by two tones at $-29.7 \mathrm{dBm}$ each, spaced $10 \mathrm{MHz}$ apart with a center frequency of $1.9 \mathrm{GHz} . m 1=8.8 \mathrm{dBm}, m 2=9.1 \mathrm{dBm}, m 3=-21.9 \mathrm{dBm}$ and $m 4=-18.9 \mathrm{dBm}$. The rest of the IM components have been excluded for clarity
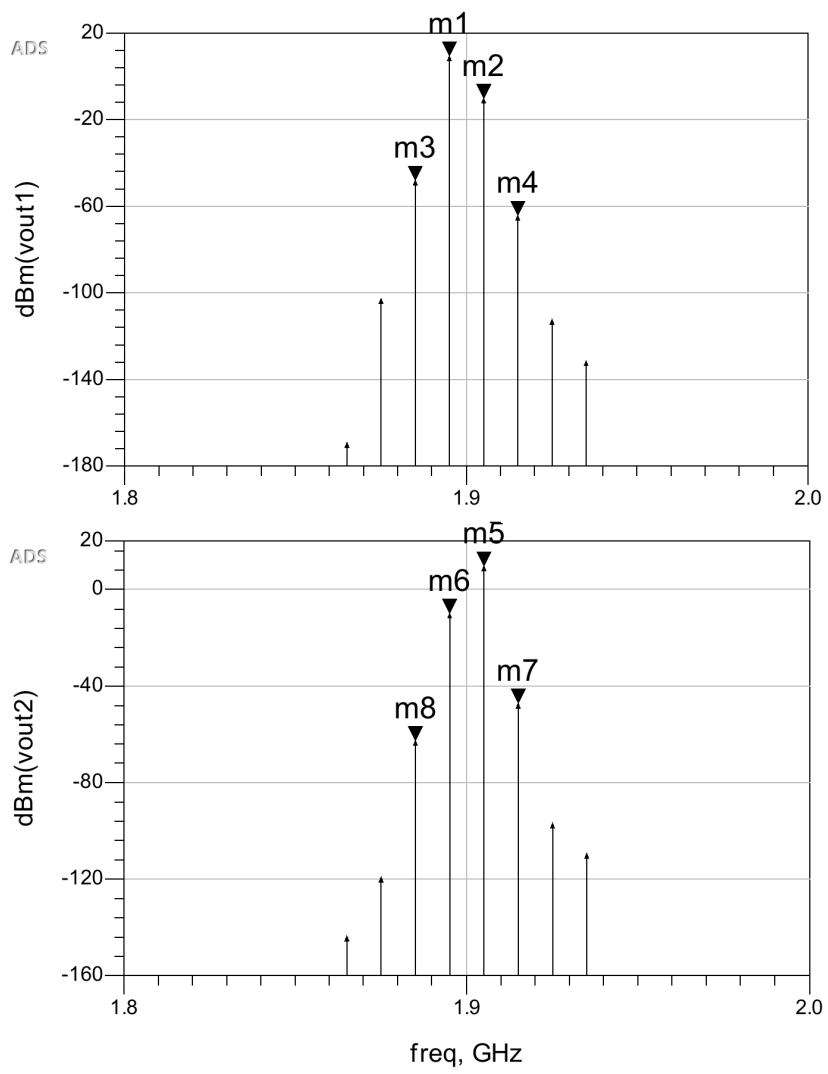

Fig. 9. PA output power spectra for an interleaved dipole antenna pair. Each $\mathrm{PA}$ is driven by a $-29.7 \mathrm{dBm}$ tone, both tones are spaced $10 \mathrm{MHz}$ apart with a center frequency of $1.9 \mathrm{GHz} . m 1=9.3 \mathrm{dBm}, m 2=-10.3 \mathrm{dBm}$, $m 3=-48.1 \mathrm{dBm}, m 4=-64.3 \mathrm{dBm}, m 5=9.7 \mathrm{dBm}, m 6=-9.9 \mathrm{dBm}$, $m 7=-47.3 \mathrm{dBm}$ and $m 8=-62.7 \mathrm{dBm}$. The other IM components are below $-160 \mathrm{dBm}$. 
PA. In combination with the low output impedance of the PA, this produces a weaker reverse IMD than its single dipole counterpart. The spurious levels for the single interleaved pair are around $-56.9 \mathrm{dBc}$, an improvement of about $28.8 \mathrm{~dB}$.

The regular $3 \times 3$ array is simulated for three different scan angle configurations in the $u$ direction. The scan angles for the two tones are defined as $u_{01}$ and $u_{02}$. Table I (a) shows the strongest spurious levels of all the elements for each arrangement. The table is organized such that each spurious signal value matches the location of each $\lambda / 2$ dipole within the array and an indexing scheme is added for clarity. First, both tones transmit in broadside, that is $\left(u_{01}, u_{02}\right)=\left(0^{\circ}, 0^{\circ}\right)$. The spurious level varies from -26 to $-11 \mathrm{dBc}$, depending on the position of the element. The center, or $(2,2)$, element experiences the strongest coupling, causing strong reverse IMD of $\Delta=-12.8 \mathrm{dBc}$, that wastes significant power in the IM3 components. The edge and side elements experience the least amount of coupling since they are the furthest away from the rest of the elements, resulting in a spectral behavior similar to that of Fig. 8. The array is next simulated with both tones steered in the same direction, chosen as $\left(u_{01}, u_{02}\right)=\left(15^{\circ}, 15^{\circ}\right)$. The spurious levels range from -24.3 to $-16.6 \mathrm{dBc}$, depending on the position of the element. This is an improvement for the center elements and a minor deterioration for the edge elements. We consider the final case where each tone is steered at a different angle such that $\left(u_{01}, u_{02}\right)=\left(-15^{\circ}, 15^{\circ}\right)$. The spurious levels of the regular array range from -17.8 to $-12.2 \mathrm{dBc}$, depending on the position of the element. In this configuration, the overall performance of the regular array is by far the worst. The center and middle edge elements have spurious levels almost as high as in the broadside simulation. The center element has a spurious level of $-12.7 \mathrm{dBc}$, while the top and bottom rows of elements experience an increase of nearly $10 \mathrm{~dB}$ compared to the broadside simulation. This behavior is in agreement with the formulation of (3), showing that the input impedance of each $\lambda / 2$ dipole within the array is dependent on the phase offsets for a given scan angle.

Next, we consider the performance of the interleaved $3 \times 3$ array. The array is simulated for the same scan angle configurations as before and the strongest spurious levels of all the elements for each arrangement are shown in Table I (b). Again, the table is organized such that each spurious level matches the location of each antenna within the array. The indexing scheme is used to group the elements in pairs for simpler comparison with Table I (a). For example the center pair of the interleaved array consists of both center elements of the regular arrays that comprise it. The array is first simulated in broadside operation with $\left(u_{01}, u_{02}\right)=\left(0^{\circ}, 0^{\circ}\right)$. Similar to the regular array, the edge elements of the interleaved array experience the weakest coupling. However, the similarities end here. The $(1,1)$ pair has spurious levels of about $\Delta_{a}=-52.3$ $\mathrm{dBc}$ and $\Delta_{b}=-47.2 \mathrm{dBc}$, respectively. The $(2,2)$, or middle, element pair has spurious levels of $\Delta_{a}=-27.2 \mathrm{dBc}$ and $\Delta_{b}=-29.7 \mathrm{dBc}$, respectively. This results in an overall IMD reduction of at least $13 \mathrm{~dB}$ compared to the regular array. In the case of the center elements of both arrays, the interleaved pair has at least $-16.9 \mathrm{~dB}$ lower spurious level than its single element counterpart. The strongest spurious level occurs at the $(2,3)$ pair with $\Delta_{a}=-24.5 \mathrm{dBc}$. Next, the array is simulated with both tones steered in $\left(u_{01}, u_{02}\right)=\left(15^{\circ}, 15^{\circ}\right)$. The overall IMD performance of the interleaved array is improved compared to the the broadside case, with some spurious levels decreasing by as much as $13 \mathrm{~dB}$, while others remain almost unchanged. Comparing with the regular array, the interleaved array has at least $-10.4 \mathrm{~dB}$ lower spurious level. The center element pair of the interleaved array has nearly $-13 \mathrm{~dB}$ lower spurious level than the center element of the regular array. The strongest spurious level again occurs at the $(2,3)$ pair having $\Delta_{a}=-27 \mathrm{dBc}$. Lastly, both tones are steered in the different directions $\left(u_{01}, u_{02}\right)=\left(-15^{\circ}, 15^{\circ}\right)$. The spurious levels of the interleaved array range from roughly -59 to $-39 \mathrm{dBc}$, depending on the element position. In this configuration, the center pair of elements have spurious levels of $\Delta_{a}=-44.3$ and $\Delta_{b}=-43.8 \mathrm{dBc}$, respectively. It is worth noting that while the performance of the regular array reduces to its broadside configuration as both tones are steered in different directions, the performance of the interleaved array improves. As before, the $(2,3)$ pair has the strongest spurious level with $\Delta_{a}=-38.7 \mathrm{dBc}$. Regarding the center elements, the interleaved array has a spurious level reduction over its regular counterpart of about $31 \mathrm{~dB}$.

The interleaved array concept enables improved power efficiency and better linear operation with the same antenna aperture. Doubling the number of PAs allows us to achieve better spectral performance with the same amount of RF input power as before. The improved power efficiency can be beneficial in systems based on constant-envelope signals by lowering the break-down margin requirements, while the better linearity can benefit systems based on modulated tones, such as QAM-modulated or MIMO systems by allowing them to operate at much lower back-off ratios. Finally, this concept also opens up the possibility to use less linear but more efficient PAs.

\section{CONCLUSIONS}

PA nonlinearity is a limiting factor in RF transmission systems, causing IMD and lowering the power and spectral efficiency. The problem is further exacerbated in phased array systems where the intermodulation components not only pollute the spectrum, but their frequency orders cause the array to transmit in unwanted directions. The densely interleaved array concept introduced in this paper occupies virtually the same area as a regular planar array while the spurious signal levels of the center elements are between $13 \mathrm{~dB}$ and $31 \mathrm{~dB}$ lower than those of the center element of the regular planar array with a PA operating at $\mathrm{P} 1 \mathrm{~dB}$, depending on the steering angles of the tones. Such an improvement in power and spectral efficiency can lead to lowering the break-down margin requirements and back-off ratios of QAM-modulated or MIMO systems.

The algorithm for computing the mutual coupling $S$ matrices is based on the closed form I-EMF method and the 
TABLE I

PERFORMANCE OF THE INTERLEAVED PLANAR ARRAY FOR DIFFERENT SCAN ANGLE CONFIGURATIONS. BOTH STRONGEST SPURIOUS LEVELS, INDICATED AS $\Delta_{n 1}$ AND $\Delta_{n 2}$, ARE SHOWN IN DBC.

\begin{tabular}{clcc}
\multicolumn{5}{c}{ (a) Regular Array } \\
Element & 1 & 2 & 3 \\
\hline \multicolumn{4}{c}{$\left(u_{01}, u_{02}\right)=\left(0^{\circ}, 0^{\circ}\right)$} \\
\hline 1 & -26.9 & -26.6 & -26.9 \\
2 & -11.1 & -12.8 & -11.1 \\
3 & -26.9 & -26.6 & -26.9 \\
\hline \multicolumn{4}{c}{$\left(u_{01}, u_{02}\right)=\left(15^{\circ}, 15^{\circ}\right)$} \\
\hline 1 & -21.8 & -21.1 & -21.8 \\
2 & -16.6 & -17.5 & -16.6 \\
3 & -22.2 & -24.3 & -22.2 \\
\hline \multicolumn{4}{c}{$\left(u_{01}, u_{02}\right)=\left(-15^{\circ}, 15^{\circ}\right)$} \\
\hline 1 & -16.9 & -17.6 & -16.9 \\
2 & -12.2 & -12.7 & -12.2 \\
3 & -17.1 & -17.8 & -17.1
\end{tabular}

assumption that the mutual impedance of an array can be constructed from a linear combination of isolated dipole pairs. The approximate nature of the algorithm necessitates increased inter-element spacings in the $v$ direction for reliable results. Future work will focus on developing more accurate closed form array mutual coupling algorithms.

\section{REFERENCES}

[1] H. Qiu, Y. Hu, Q. Hu, X. Zhu, Z. Yang, and B. Li, "Optimization of intermodulation distortion for a Ku-band helix TWT," in 2018 IEEE International Vacuum Electronics Conference (IVEC), Principal Monterey, CA, USA, April 2018, pp. 149-150.

[2] W. Tsai, C. Liou, Z. Peng, and S. Mao, "Intermodulation Distortion Analysis for Power Amplifier with Various Collector Voltages," in 2018 IEEE International Symposium on Radio-Frequency Integration Technology (RFIT), Melbourne, Australia, Aug 2018, pp. 1-3.

[3] Z. El-Khatib, L. MacEachern, and S. A. Mahmoud, "Improvement of carrier power to third-order intermodulation distortion power ratio in CMOS distributed amplifiers," in 2008 International Conference on Microelectronics, Piscataway, NJ, USA, Dec 2008, pp. 98-101.

[4] R. Negra, F. M. Ghannouchi, and W. Bachtold, "Compact devicelevel linearisation technique using a reduced complexity derivative superposition approach," in 2006 Asia-Pacific Microwave Conference, Pacifico Yokohama, Yokohama, Japan, Dec 2006, pp. 1739-1742.

[5] S. P. Stapleton, "Adaptive FeedForward Linearization For RF Power Amplifiers," in 55th ARFTG Conference Digest, vol. 37, Boston, USA, June 2000, pp. 1-7.

[6] F. H. Gregorio, G. J. Gonzlez, J. Cousseau, T. Riihonen, and R. Wichman, "Predistortion for power amplifier linearization in full-duplex transceivers without extra RF chain," in 2017 IEEE International Conference on Acoustics, Speech and Signal Processing (ICASSP), New Orleans, LA, USA, March 2017, pp. 6563-6567.

[7] G. J. Mazzaro, K. G. Gard, and M. B. Steer, "Low distortion amplification of multisine signals using a time-frequency technique," in 2009 IEEE MTT-S International Microwave Symposium Digest, Boston, USA, June 2009, pp. 901-904.

[8] A. Katz, D. McGee, C. Brinton, and J. Qiu, "Sensitivity and mitigation of Reverse IMD in power amplifiers," in 2011 IEEE Topical Conference on Power Amplifiers for Wireless and Radio Applications, Phoenix, Arizona, USA, Jan 2011, pp. 53-56.

[9] E. Hallén, Theoretical Investigations Into the Transmitting and Receiving Qualities of Antennae, ser. Acta Universitatis Upsaliensis: Nova acta Regiae Societatis Scientiarum Upsaliensis. Almqvist \& Wiksells, 1938.

[10] H. Pocklington, "Electrical oscillations in wires," Cambridge Philosophical Society - Proceedings, pp. 324-332, 101897.

[11] D. B. Davidson, An overview of computational electromagnetics for $R F$ and microwave applications, 2nd ed. Cambridge University Press, 2010, p. 131.

[12] K. Aniserowicz, "Comparison of different numerical methods for solving boundary-value problems in electromagnetics," IEEE Transactions on Education, vol. 47, no. 2, pp. 241-246, May 2004.

\begin{tabular}{cccc}
\multicolumn{4}{c}{ (b) Interleaved Array } \\
Pair & 1 & 2 & 3 \\
\hline \multicolumn{4}{c}{$\left(u_{01}, u_{02}\right)=\left(0^{\circ}, 0^{\circ}\right)$} \\
\hline 1 & $(-52.3,-47.2)$ & $(-48.1,-41.7)$ & $(-47.6,-45.0)$ \\
2 & $(-41.2,-27.3)$ & $(-27.2,-29.7)$ & $(-24.5,-42.2)$ \\
3 & $(-50.4,-50.0)$ & $(-46.0,-47.1)$ & $(-46.8,-49.9)$ \\
\hline \multicolumn{4}{c}{$\left(u_{01}, u_{02}\right)=\left(15^{\circ}, 15^{\circ}\right)$} \\
\hline 1 & $(-53.8,-46.1)$ & $(-47.4,-42.3)$ & $(-46.2,-46.3)$ \\
2 & $(-48.4,-40.0)$ & $(-30.0,-39.1)$ & $(-27.0,-43.5)$ \\
3 & $(-55.3,-55.0)$ & $(-54.8,-48.8)$ & $(-50.7,-54.3)$ \\
\hline \multicolumn{4}{c}{$\left(u_{01}, u_{02}\right)=\left(-15^{\circ}, 15^{\circ}\right)$} \\
\hline 1 & $(-58.6,-39.4)$ & $(-53.6,-42.8)$ & $(-53.1,-47.8)$ \\
2 & $(-48.6,-40.0)$ & $(-44.3,-43.8)$ & $(-38.7,-47.8)$ \\
3 & $(-47.2,-51.9)$ & $(-42.7,-53.2)$ & $(-40.8,-56.5)$
\end{tabular}

[13] M. N. O. Sadiku and A. F. Peterson, "A comparison of numerical methods for computing electromagnetic fields," in IEEE Proceedings on Southeastcon, April 1990, pp. 42-47 vol.1.

[14] H. King, "Mutual impedance of unequal length antennas in echelon," IRE Transactions on Antennas and Propagation, vol. 5, no. 3, pp. 306313, 71957.

[15] R. W. P. King, "Electric fields and vector potentials of thin cylindrical antennas," IEEE Transactions on Antennas and Propagation, vol. 38, no. 9, pp. 1456-1461, Sep. 1990.

[16] T. Katagi, H. Ohmine, H. Miyashita, and K. Nishimoto, "Analysis of Mutual Coupling Between Dipole Antennas Using Simultaneous Integral Equations With Exact Kernels and Finite Gap Feeds," IEEE Transactions on Antennas and Propagation, vol. 64, no. 5, pp. 1979-1984, May 2016.

[17] J. Kraus and R. Marhefka, Antennas for all applications, ser. McGrawHill series in electrical engineering. McGraw-Hill, 2002, ch. 10, p. 413.

[18] C. A. Balanis, Antenna theory: analysis and design. Wiley-Interscience, 2005 , ch. 8 , p. 433.

[19] R. L. Haupt, Mutual Coupling. IEEE, 2010, p. 342. [Online]. Available: https://ieeexplore.ieee.org/document/5599479

[20] P. S. Carter, "Circuit Relations in Radiating Systems and Applications to Antenna Problems," Proceedings of the Institute of Radio Engineers, vol. 20, no. 6, pp. 1004-1041, 61932.

[21] T. Reveyrand, "Multiport conversions between S, Z, Y, h, ABCD, and T parameters," in 2018 International Workshop on Integrated Nonlinear Microwave and Millimetre-wave Circuits (INMMIC), Brive La Gaillarde, France, July 2018, pp. 1-3.

[22] A. Voors. (2015) NEC based antenna modeler and optimizer. [Online]. Available: https://www.qsl.net/4nec2/

[23] R. M. Henderson. (2009) LAB 7: Harmonic Balance Simulations. [Online]. Available: https://www.utdallas.edu/ rmh072000/Site/Software_ and_Links_files/7B_lab.pdf 